\title{
"Nebelsprech" \\ Sprechen in der parlamentarischen Demokratie
}

\author{
Martin Haase (Bamberg)
}

\begin{abstract}
"Fogspeech" is a variant of Orwellian Newspeak typical of parliamentary Democracy: As it is important in a Democracy to find vast support for political projects, linguistic strategies from advertising are used, esp. positively connotated words, positive framing, hyperbolic speech. At the same time, politicians who want to avoid direct responsibility tend to use nebular expressions, set phrases, passive and impersonal constructions etc. A number of examples are discussed.
\end{abstract}

\section{Sprache bringt es an den Tag}

Von Victor Klemperer (1947: 312) stammt die denkwürdige Feststellung:

Was jemand willentlich verbergen will, sei es vor anderen, sei es vor sich selber, auch was er unbewusst in sich trägt: Die Sprache bringt es an den Tag.

(Klemperer 1947: 312)

Das klingt paradox, denn Ausdrucksmittel, die etwas salopp in Anlehnung an das Newspeak aus Orwells (1949) 1984 als "Nebelsprech" bezeichnet werden können, sollen gleichzeitig verbergen und etwas an den Tag bringen: Es handelt sich dabei vor allem um sprachliche Füllsel (Pleonasmen), die im jeweiligen Kontext nichts zur Bedeutung eines Textes beitragen, sondern einer Aussage zum Beispiel Nachdruck verleihen sollen, den die Aussage gar nicht benötigen würde, wenn sie ernstgemeint wäre. So hieß es bei den Koalitionsverhandlungen zwischen SPD und Grünen zum Weiterbau der A100 in Berlin:

Das Projekt des 16. Bauabschnitts der BAB 100 wird nicht grundsätzlich aufgegeben. Die Koalition setzt sich aber aktiv und ernsthaft dafür ein, dass eine Umwidmung der Bundesmittel ermöglicht wird.

(Die Grünen Berlin 2011: Zeile 112-113)

Die Adverbien aktiv und ernsthaft haben hier eine entlarvende Wirkung, denn ein passiver und scherzhafter Einsatz für eine Forderung ist ja gar nicht vorstellbar. In der Rhetorik spricht man in diesem Zusammenhang von einer Hyperbel, die allerdings im vorliegenden Fall misslungen ist, denn die hyperbolische Steigerung legt nahe, dass mit Aktivitäten in diesem Zusammenhang möglicherweise nicht zu rechnen ist. Und so war dann bald auch nicht mehr mit der rot-grünen Koalition zu rechnen. 
Der Versuch, etwas sprachlich zu vertuschen (hier das Aufgeben eines zentralen Wahlkampfversprechens der Grünen), wirkt gerade entlarvend, weil bei einer genauen sprachlichen Analyse deutlich wird, dass bestimmte Ausdrucksmittel nicht zusammenpassen. Das gilt für alle sprachlichen Mittel der Verschleierung, die im Folgenden genauer vorgestellt werden.

\section{$2 \quad$ Framing}

Framing ist ein für die Sprache der Politik wichtiges Phänomen: Ein Wort erweckt bestimmte Bedeutungsassoziationen, einen semantischen Kontext, der das durch das Wort vermittelte Konzept als mehr oder weniger wünschenswert erscheinen lässt. Der Entdecker dieses Phänomens George Lakoff (2014) illustriert es mit zahlreichen Beispielen: so änderte die American Association of Trial Lawyers ihren Namen in American Association of Justice, weil justice nicht nur "Justiz", sondern auch "Gerechtigkeit" bedeutet. Im Folgenden zwei Beispiele aus der deutschen politischen Diskussion.

\subsection{Rettungsschirm}

Ein besonders interessanter Fall ist der Rettungsschirm. Hier das erste Zitat, das Kai Biermann und ich für dieses Wort im Zusammenhang mit der Finanzkrise gefunden haben, als wir für unseren Blog neusprech.org recherchiert haben:

Die IKB gibt eine Gewinnwarnung heraus und teilt mit, dass Großaktionär KfW bürgen muss. Details sickern durch: Die Kreditwirtschaft rettet die IKB per milliardenschwerem Rettungsschirm.

(Financial Times Deutschland, 13.08.2007 zit. nach Gruner/Jahr 2012)

Es scheint sich also um eine Schöpfung der Pressesprache zu handeln, bevor es auch in der Politik Verwendung findet:

Die Euro-Schuldenkrise zwingt uns zu weitreichenden Maßnahmen. Am 29. September 2011 haben wir im Bundestag grünes Licht für den erweiterten Rettungsschirm EFSF gegeben.

(CDU/CSU-Fraktion 2011)

Die Quelle ist eine Publikation der CDU/CSU-Fraktion. So dringt der Rettungsschirm allmählich in die offizielle politische Terminologie ein und wird schließlich auch in einem Text des Bundestages verwendet, und zwar anstelle des etwas sperrigen Terminus "europäischer Stabilitätsmechanismus (ESM)":

Breite Mehrheit für Fiskalpakt und Rettungsschirm: Der Bundestag hat am Freitag, 29. Juni 2012, dem Fiskalpakt zugestimmt. Die erforderliche Zweidrittelmehrheit wurde damit deutlich übertroffen. Mit 491 Ja-Stimmen bei 111 Nein-Stimmen und sechs Enthaltungen stimmte das Parlament dem Gesetzentwurf von Union und FDP zum Vertrag vom 2. März 2012 über Stabilität, Koordinierung und Steuerung in der Wirtschafts- und Währungsunion zu.

(Deutscher Bundestag 2012)

Aus der Pressedatenbank des Verlagshauses Gruner und Jahr (2012) ergibt sich, in wie vielen Artikeln in den Jahren 2007 bis 2012 das Wort Rettungsschirm Verwendung findet (Tabelle 1). 


\begin{tabular}{|l|l|}
\hline Jahr & Artikel \\
\hline 2007 & 6 \\
\hline 2008 & 615 \\
\hline 2009 & 1.260 \\
\hline 2010 & 2.348 \\
\hline 2011 & 8.753 \\
\hline 2012 & 6.646 \\
\hline
\end{tabular}

Tabelle 1: Artikel in deutschen Zeitungen und Zeitschriften, in denen Rettungsschirm verwendet wird, ermittelt aus Gruner und Jahr (2012), 380 Quellen

Bei frühen Belegen ist mit Rettungsschirm noch ein Hilfsfallschirm gemeint; wenn jedoch die metaphorische Bedeutung nach 2007 in den Vordergrund tritt, wird nicht mehr an den Hilfsfallschirm gedacht, sondern an einen Regenschirm, wie eine Abbildung aus einem Artikel des Bundesfinanzministeriums zeigt (Bundesministerium der Finanzen 2009). Sprachwissenschaftlich ist es ein interessantes Beispiel dafür, wie sich die Metaphorik eines nicht wörtlich verwendeten Wortes verändern kann.

Ein Regenschirm ist ein viel angenehmeres Bild als ein Hilfsfallschirm, dessen Funktion darin besteht, einen nicht-funktionierenden Fallschirm zu kompensieren. Ein Regenschirm bietet schirmenden Schutz. Diese positive Assoziation mag nicht nur für die Popularität des Ausdrucks gesorgt haben, sondern auch in Zusammenhang stehen mit der erfolgreichen Durchsetzung des ESM in Deutschland im Vergleich zu der wesentlich kritischeren Auseinandersetzung darüber in den meisten anderen europäischen Ländern.

\subsection{Nacktscanner}

Das Wort Nacktscanner ist wahrscheinlich auch eine Presseschöpfung, die allerdings von Innenpolitikern nicht besonders wohlwollend aufgenommen wurde. Stattdessen wurden andere Wörter präferiert:

Einen Nacktscanner wird es mit mir nicht geben. Körperscanner der zweiten Generation kann es dagegen sehr wohl geben.

(de Maiziere 2010: 1226)

Nacktscanner hat zu negative Assoziationen, da dieses Wort deutlich macht, wie sehr diese Geräte die Intimsphäre der gescannten Personen verletzen. Abbildung 1 zeigt jedoch sehr schön, dass das konkurrierende Wort Körperscanner gegenüber dem negativ konnotierten Nacktscanner keine Chance hatte. Diese Abbildung vergleicht die Vorkommen der beiden Wörter über mehrere Jahre, wobei der höchste Ausschlag der Verwendung von Nacktscanner die $100 \%$-Marke definiert. 


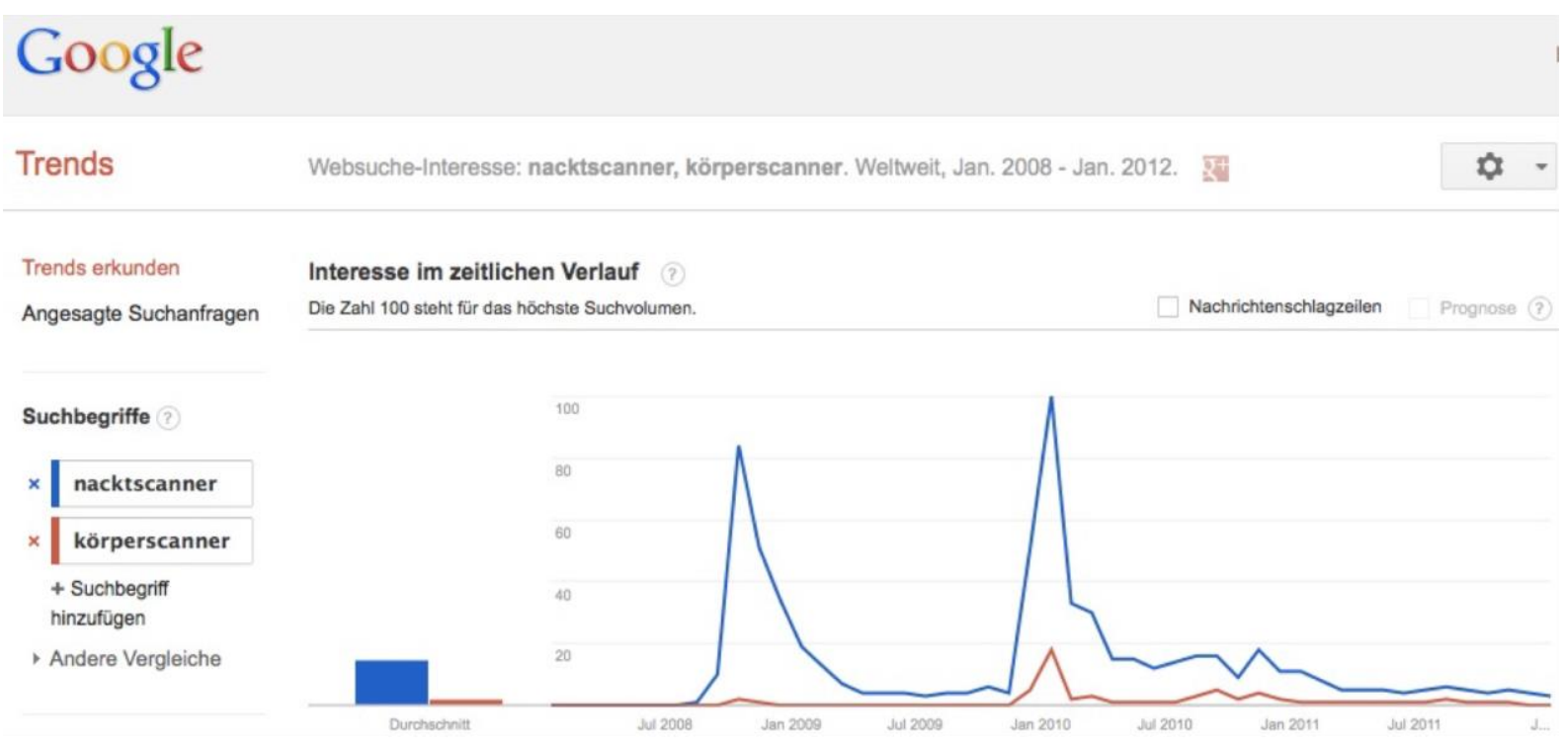

Abbildung 1: Nacktscanner vs. Körperscanner. Quelle: Google Trends

Hier eine Liste der verwendeten Wörter für diese Art von Personenscannern, wobei immer angegeben ist, in welchem Bereich bzw. von wem das jeweilige Wort verwendet wird:

- Terahertzscanner - technisch

- Millimeterwellen-Scanner - technisch

- Körperscanner - Bundesinnenministerium

- Sicherheitsscanner - EU-Kommission

- Nacktscanner - Medien

- Ganzkörperscanner - Bundesinnenministerium/Medien

- Bodyscanner/Full-Body-Scanner - englisch

Tabelle 2 gibt die Zahl der Zeitungs- bzw. Zeitschriftenartikel an, in denen sie jeweils verwendet werden.

\begin{tabular}{|l|l|}
\hline Begriff & Artikel \\
\hline Nacktscanner & 554 \\
\hline Körperscanner & 408 \\
\hline Ganzkörperscanner & 71 \\
\hline Bodyscanner & 62 \\
\hline Sicherheitsscanner & 10 \\
\hline Terahertzscanner & 3 \\
\hline
\end{tabular}

Tabelle 2: Zahl der Artikel in deutschen Medien, in denen die jeweilige Bezeichnung zwischen 2008 und 2012 erwähnt wurden (380 Quellen), ermittelt aus Gruner/Jahr 2012

Deutlich zu erkennen ist die starke Präferenz von Nacktscanner, womit eigentlich klar ist, dass die Innenpolitiker, die diese Geräte durchsetzen wollen, sprachlich und damit wohl auch in der Sache gescheitert sind. 
Das Beispiel zeigt sehr schön einen wichtigen Aspekt des Framings: Es gibt keine neutralen (Schlüssel-) Begriffe in der Sprache der Politik: Gerade wenn jemand den Präzisismus Ganzkörperscanner oder den Technizismus Terahertzscanner verwendet, ist das verräterisch, denn damit erweist sich der Verwender als Befürworter dieser Geräte, da ein Gegner solche euphemistischen Wortschöpfungen vermeiden würde - zugunsten des "griffigen" Dysphemismus Nacktscanner, den ein Befürworter typischerweise zu vermeiden sucht.

Es wird deutlich, dass eine parlamentarische Demokratie besonders anfällig ist für die hier beschriebene Variante von "Neusprech": Politische Vorhaben benötigen eine breite Unterstützung, um sich durchzusetzen. Es muss also für sie geworben werden, im Extremfall wie für ein Produkt, insbesondere wenn der Nutzen eines Vorhabens - wie im vorliegenden Fall fragwürdig ist. So kommt es zur Verwendung von sprachlichen Strategien, die sonst eher aus der Werbung bekannt sind, wie zum Beispiel beim Rückgriff auf positiv konnotierte Wörter.

\section{Grammatischer Nebel}

Neben den lexikalischen Vernebelungsstrategien, die in Abschnitt 1 beschrieben wurden, gibt es auch grammatische Mechanismen (vgl. Biermann/Haase 2012).

\subsection{Guttenberg-Passiv}

Während Aktiv und Passiv im Abschnitt 1 lexikalisch verwendet wurden, hat die grammatische Kategorie Passiv ebenfalls großes Vernebelungspotential, denn eine Funktion des Passivs ist es, die Nennung eines Handelnden (Agens) zu unterdrücken. Dies ist zwar nur eine Funktion des Passivs, die jedoch von einem Politiker auffällig häufig verwendet wird: Am 18.02.2011 gab Karl-Theodor zu Guttenberg (2011) die folgende Erklärung ab:

Für diese Stellungnahme bedurfte es keiner Aufforderung und sie gab es auch nicht. Meine von mir verfasste Dissertation ist kein Plagiat, und den Vorwurf weise ich mit allem Nachdruck von mir. Sie ist über etwa sieben Jahre neben meiner Berufs- und Abgeordnetentätigkeit als junger Familienvater in mühevollster Kleinarbeit entstanden, und sie enthält fraglos Fehler. Und über jeden einzelnen dieser Fehler bin ich selbst am unglücklichsten.

Es wurde allerdings zu keinem Zeitpunkt bewusst getäuscht oder bewusst die Urheberschaft nicht kenntlich gemacht. [...]

(Guttenberg 2011)

Offenbar möchte zu Guttenberg hier nicht sagen, wer derjenige ist, der getäuscht hat oder die Urheberschaft der plagiierten Texte nicht kenntlich gemacht hat. So bleibt offen, ob er selbst dies war oder ein Ghostwriter, dessen Existenz allerdings durch die ebenfalls auffällige Partizip-Passiv-Konstruktion "Meine von mir verfasste Dissertation" ausgeschlossen wird. Beide Formulierungen sind im Sinne Klemperers entlarvend: "Meine von mir verfasste Dissertation" enthält eine ungewöhnliche Dopplung: Zwar könnte "meine Dissertation" angesichts der Plagiatsvorwürfe durchaus bedeuten, dass zu Guttenberg sie nicht selbst verfasst haben müsste, aber mit "die von mir verfasste Dissertation" wäre ja schon klar gewesen, wer der Verfasser oder Plagiator ist. Die Wiederholung des Adverbs bewusst - in einer Passivkonstruktion unpassend - betont diesen Umstand sehr, wobei sich die Frage stellt, wie man unbewusst täuschen kann. Erschwerend kommt noch hinzu, dass das Adverbial zu keinem Zeitpunkt sich auf den ganzen Satz bezieht, wodurch eine doppelte Verneinung unumgänglich ist: "Es wurde 
allerdings zu keinem Zeitpunkt [...] bewusst die Urheberschaft nicht kenntlich gemacht." Solche verqueren Konstruktionen bringen es an den Tag, dass hier etwas zu hinterfragen ist, dass eben mehr als nur "Fehler" vorliegen. Die Hyperbeln tun ein Übriges: Welche "Kleinarbeit" ist denn nicht mühevoll? Auf die "Kleinarbeit" wird noch zurückzukommen sein.

Bei seinem Comeback sagt zu Guttenberg:

Die sorgfältige Detailarbeit, gerade das korrekte Einarbeiten und Zitieren fremder Quellen, ist wiederholt unterblieben.

(Di Lorenzo 2011)

Aus der "Kleinarbeit" ist nun "Detailarbeit" geworden, die nun aber "wiederholt unterblieben" ist. Auch hier liegt wieder eine Konstruktion vor, die der Passivkonstruktion zumindest ähnelt: ist unterblieben ist ein Resultativ-Perfekt, das mit dem sogenannten Zustandspassiv eng verwandt ist: auch hier muss kein Agens genannt werden.

$\mathrm{Zu}$ Guttenberg wechselt in seinem Interview die Strategie:

Es steht völlig außer Frage, dass ich einen auch für mich selbst ungeheuerlichen Fehler begangen habe, den ich auch von Herzen bedauere. Das ist in dieser sehr hektischen Zeit damals auch ein Stück weit untergegangen. Ebenso, wie man sich damals bereits entschuldigt hat.

(Ebd.)

Hier wird jetzt mit man eine unpersönliche Konstruktion verwendet, die so auffällig ist, dass Giovanni Di Lorenzo seinen Gesprächspartner gleich darauf anspricht. Dieser entgegnet:

Das "man" soll auch keine Distanzierung bedeuten. Es ist ein anerzogener Sprachgebrauch, der sich bei mir wahrscheinlich über die Jahre hinweg eingeschliffen hat, der eine gewisse Form von Zurückhaltung zum Ausdruck bringen soll und den man zu Recht kritisieren kann.

(Ebd.)

Dann geht es weiter im Passiv: Zweimal verwendet zu Guttenberg die folgende Formulierung:

Der Fehler war bereits relativ früh angelegt.

(Ebd.)

Es handelt sich um ein Zustandspassiv, das noch dazu im Präteritum verwendet wird. Damit ist nicht nur kein Agens vorhanden, sondern durch die Vergangenheit noch weiter der Kontrolle durch einen Handelnden entzogen. Doch damit nicht genug! Weiter heißt es in demselben Interview:

Aber in diesem unglaublichen Wust von selbst geschriebenen und fremden Fragmenten hätten die fremden Fragmente eben mit Quellenangaben sauber gekennzeichnet werden müssen.

(Ebd.)

Das ist zwar ein Eingeständnis, aber es handelt sich wieder um selbst geschriebene Fragmente (Partizip Passiv) und es wird auch nicht deutlich, wer die fremden Fragmente hätte kennzeichnen müssen - ein Ghostwriter, jemand vom wissenschaftlichen Dienst oder vielleicht doch der Kandidat, der eine "summa-cum-laude-Dissertation" vorlegen will.

Auf die Frage, ob zu Guttenberg nicht daran gedacht habe, dass Texte mit Suchmaschinen als Plagiate identifiziert werden könnten, kommt wieder ein Passiv: 
$\mathrm{Na}$ ja, in den Jahren 2005 und 2006, als die Arbeit abgeschlossen wurde, war das sehr wohl bekannt.

(Ebd.)

Es passt so gar nicht zu Guttenbergs sonstigem Auftreten, dass er sich so zurücknimmt. Zwei Erklärungen liegen nahe: dass er die Plagiate nicht selbst oder zumindest nicht allein gesammelt hat und dass er selbst nicht als schuldhaft Handelnder dastehen will. Selbstverständlich schließt die eine Erklärung die andere nicht aus.

\subsection{Merkel-Wir}

Abbildung 2 zeigt eine Wortwolke für Angela Merkels (2012a, 2012b) Rede auf dem CDUParteitag in Hannover 2012.

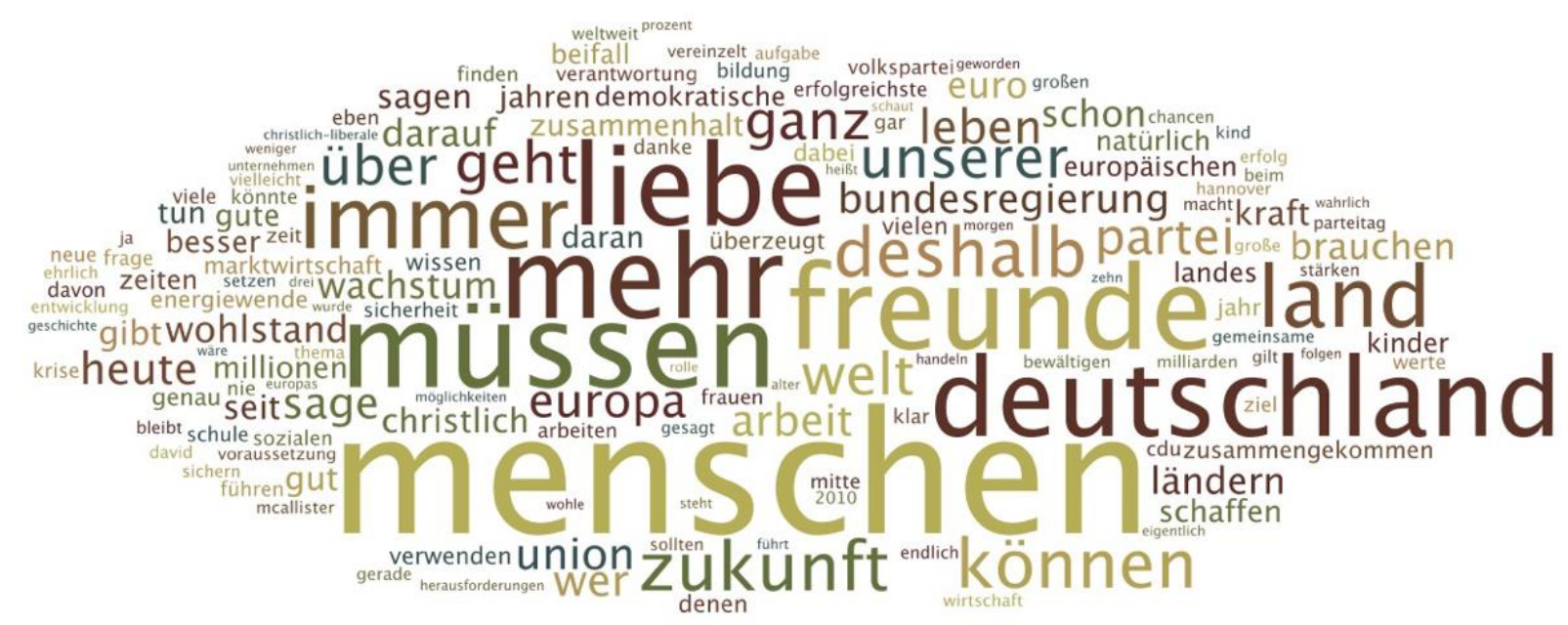

Abbildung 2: Wortwolke: nur Inhaltswörter in Merkel (2012a), erstellt mit www.wordle.net

Hier geht es nur um die Häufigkeit von Inhaltswörtern: Je größer ein Wort in der Wortwolke ist, umso häufiger kommt es vor. Auf diese Weise lässt sich auf einen Blick erkennen, welche Themen in der Rede wichtig sind. Wobei es auch zu Irreführungen kommen kann: Liebe ist kein wichtiges Thema in Angela Merkels Rede, aber die häufige Anrede liebe Freunde führt natürlich dazu, dass diese Wörter besonders häufig sind.

In der Regel werden in Wortwolken Funktionswörter weggelassen, da sonst immer dieselben kurzen Wörter hervortreten. Bei Angela Merkel ist es jedoch durchaus sinnvoll, auch eine Wortwolke unter Einschluss der Funktionswörter anzufertigen, weil sich etwas Untypisches abzeichnet, wie Abbildung 3 zeigt: 


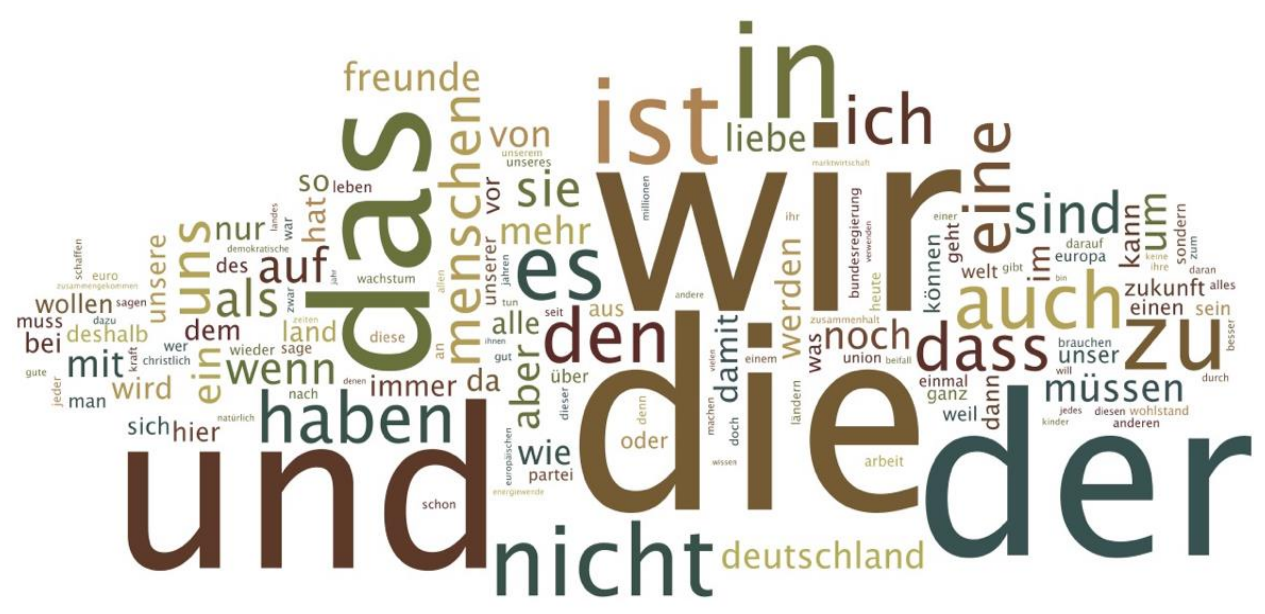

Abbildung 3: Wortwolke: alle Wörter aus Merkel (2012a), erstellt mit www.wordle.net

Das Personalpronomen wir, das gar nicht das häufigste Funktionswort im Deutschen ist, tritt besonders hervor. Es ist sogar bei Merkel das häufigste Wort überhaupt, wie Tabelle 3 zeigt, sogar mit Abstand, wenn die Deklinationsform uns noch hinzugezählt wird.

\begin{tabular}{|l|l|}
\hline Wort & Häufigkeit \\
\hline wir & $233+52$ uns $=285$ \\
\hline die & 231 \\
\hline der & 200 \\
\hline und & 196 \\
\hline das & 149 \\
\hline ist & 106 \\
\hline
\end{tabular}

Tabelle 3: Worthäufigkeiten in Merkel (2012a), erstellt mit NLTK (Bird/Klein/Loper 2009)

Mit wir meint Angela Merkel unterschiedliche Personengruppen: die Bundesregierung bzw. die damalige CDU-CSU-FDP-Koalition, ihre Partei oder auch alle Deutschen. Dabei verändert das wir seine Extension bisweilen innerhalb eines Absatzes:

CDU, CSU und FDP haben im Vergleich zu allen anderen Konstellationen immer noch die größten Gemeinsamkeiten. Vor allem: Wir teilen gemeinsame Werte und Grundsätze. Das sind genau die Werte und Grundsätze, die wir brauchen, [...] um unsere Art zu leben und um unseren Wohlstand zu sichern.

(Merkel 2012a)

Hier bezieht sich das erste wir auf die Koalition, das nächste jedoch (zusammen mit unsere(n)) kann nur ein inklusives wir sein, dass zumindest die Hörer miteinschließt, wenn nicht sogar so etwas wie wir Deutschen gemeint ist.

Weniger inklusiv ist das wir im darauf folgenden Abschnitt ihrer Rede:

So machen wir das: nicht zu unserem Wohle, sondern zum Wohle aller Menschen in Deutschland, zum Wohle Europas. Das ist unsere Mission, das ist unsere Aufgabe. 
Es ist nicht ganz klar, ob das wir hier inklusiv oder exklusiv ist, ob also die Zuhörer eingeschlossen sind oder nicht. Auf jeden Fall wird deutlich, dass das Pronomen der ersten Person Plural im Gegensatz zur Verwendung weiter oben nicht so extensiv ist, dass alle Deutschen mit einbezogen sind.

Wie die Slogans der CDU/CSU bei den vergangenen Wahlen zeigen, ist das Pronomen wir eine Art Markenzeichen der CDU. So hieß es: "Wir in Europa" (Europawahl 2009) oder "Wir haben die Kraft" (Bundestagswahl 2009), wobei das Pronomen jeweils auf einer Deutschlandfahne gedruckt war. Die CSU warb 2013 mit Angela Merkel unter dem Slogan "unsere Kanzlerin", wobei völlig unklar bleibt, wer mit unsere hier gemeint ist. Vor diesem Hintergrund war es ungeschickt von der SPD, die Idee mit dem Pronomen der ersten Person Plural zu übernehmen, noch dazu in schlecht: Der Slogan "Das WIR entscheidet" war schwer verständlich, zumal viele nicht wussten, dass das Pronomen, das ja hier ein Substantiv ist, mit großem Anfangsbuchstaben zu schreiben ist.

In der Politikersprache changiert das Pronomen der ersten Person in Bezug auf seine Extension. Es wird fast zum Passepartout-Wort, was erklärt, warum es so häufig verwendet wird.

\subsection{Konjunktiv}

Neben der häufigen Verwendung des Pronomens der ersten Person Plural nutzt Merkel eine weitere grammatische Kategorie zur Vernebelung, nämlich den Konjunktiv II (Irrealis): So antwortet sie auf die Frage nach der Abschaltung alter Kernkraftwerke, deren Laufzeitverlängerung gerade beschlossen war:

Das wäre die Konsequenz, denn sonst wäre es kein Moratorium des von uns neu beschlossenen Gesetzes.

(Merkel 2011)

Eigentlich schließen sich die Konjunktive gegenseitig aus, denn entweder ist die Konsequenz irreal oder aber das Moratorium: Das ist die Konsequenz, denn sonst wäre es kein Moratorium bzw. Das wäre die Konsequenz, denn es ist kein Moratorium.

Und auf die Zusatzfrage, wann abgeschaltet wird:

Ich würde sagen: Wenn wir mit den Kernkraftwerksbetreibern gesprochen haben.

(Ebd.)

Hier dient der Konjunktiv II offenbar dazu, die Aussage abzumildern.

\section{$4 \quad$ Pragmatik und Stilistik}

\subsection{Präsupposition}

Besonders interessant ist ein weiteres Vernebelungsinstrument, nämlich das der Präsupposition. Was damit gemeint ist, sei an einem fiktiven Beispiel erläutert. Der folgende Satz hätte so oder so ähnlich in einer Zeitung stehen können:

Zu seiner Zeit als unbescholtener Politiker traf er sich häufiger mit dem AWD-Gründer Carsten Maschmeyer. 
Selbst wenn man den Satz verneint und das Gegenteil behauptet (zum Beispiel im Rahmen einer Gegendarstellung), bleiben bestimmte Behauptungen unverändert:

Zu seiner Zeit als unbescholtener Politiker traf er sich nicht häufiger mit dem AWD-Gründer Carsten Maschmeyer.

Immer noch gilt:

- Es fanden Treffen statt.

- Es wird behauptet, dass der Politiker ("er") seinerzeit unbescholten war.

- Durch die Zeitangabe und das Präteritum wird klar, dass "er" jetzt offensichtlich nicht mehr unbescholten ist. (Genaugenommen handelt es sich hier um eine Verknüpfung einer Präsupposition mit einer Implikatur).

Auf diese Weise gelingt es, mit Präsuppositionen in untergeordneten Satzstrukturen (in der Regel in Adverbialen) gewisse Dinge fast unmerklich zu unterstellen. Dies geschieht auch in Merkels Pressestatement zu Fukushima:

Und dennoch: Es war und es ist kein leeres Wort, wenn ich sage: Wir können nicht einfach zur Tagesordnung übergehen und die bisherige unbestrittene Sicherheit unserer kerntechnischen Anlagen zum Maßstab auch des künftigen Handelns machen, ohne dass wir infolge der jüngsten Ereignisse einmal innehalten.

(Merkel 2011)

Hier bekommen wir ganz beiläufig die "Sicherheit unserer kerntechnischer Anlagen" vermittelt. Da es sich dabei nicht um die Hauptaussage handelt, sondern um eine Präsupposition, wird diese Passage kaum hinterfragt. Ein wenig wird das "Unter-die-Weste-Jubeln" jedoch durch das Adjektiv unbestritten verhindert, denn so ein hyperbolisches Adjektiv lenkt natürlich den kritischen Geist auf die präsupponierte "Sicherheit".

\subsection{Nominalstil}

2011 beschloss die SPD (2011) die Vorratsdatenspeicherung, ohne jedoch diesen Beschluss beim Namen zu nennen. Vielmehr lautet die Überschrift wie folgt:

Datenschutz und Grundrechte stärken - Datenspeicherung begrenzen!

(SPD 2011)

Datenschutz, Grundrechte und stärken sind positiv besetzte (positiv konnotierte) Wörter, das negativ konnotierte Vorratsdatenspeicherung wird vermieden (es wird dreimal im Text verwendet, allerdings einmal im Kontext mit einer Negation und ein anderes Mal mit Bezug auf die Diskussion in CDU und FDP), dafür fällt auch das Wort Mindestspeicherung ("Neusprech" für Vorratsdatenspeicherung). Die Überschrift enthält jedoch eine Präsupposition: Wenn es (nur) darum geht, die Datenspeicherung zu begrenzen, wird die Datenspeicherung an sich gar nicht in Frage gestellt, d. h.: die grundsätzliche Ablehnung der die Grundrechte verletzenden (Vorrats-) Datenspeicherung kommt gar nicht in Betracht.

Hier noch eine weitere Passage aus dem sprachlich auffälligen Text:

Wir setzen uns auf europäischer Ebene für eine Revision der EU-Richtlinie ein. Insbesondere für die Möglichkeit auf nationaler Ebene weitere Einschränkungen regeln zu können: 
- eine Verkürzung der Speicherfristen auf deutlich unter sechs Monate. Feststellungen in der Praxis haben ergeben, dass eine Speicherdauer von drei Monaten in aller Regel ausreichend ist.

- eine Differenzierung der Speicherdauer und Zugriffsvoraussetzungen anhand der zu speichernden Datenarten hinsichtlich ihrer Eingriffsintensität.

(Ebd.)

Auch hier ist von der "Verkürzung der Speicherfristen" die Rede. Dass aus grundsätzlichen Erwägungen eine verdachtsunabhängige Speicherung von Kommunikationsdaten gar nicht erst in Frage kommen sollte, ist durch die Präsupposition ausgeschlossen. Eine Spielart des Passivs zeigt sich in der Nominalisierung Feststellungen: Hier wird nicht genannt, wer so etwas festgestellt hat. Auch der Plural trägt hier zur Nebelbildung bei.

Noch nebliger wird es dann im nächsten (verbfreien) Absatz: Zunächst ist nicht klar, ob Zugriffsvoraussetzungen auch von Differenzierung abhängig ist oder neben der Differenzierung steht. Auch der Bezug von hinsichtlich ist unklar (bezieht sich der Rest auf Datenarten, auf Zugriffsvoraussetzungen oder auf Differenzierung?). Ob ihrer das Substantiv Datenarten oder das eigentlich zu weit entfernte Zugriffsvoraussetzungen aufgreift, kann auch nicht sicher geklärt werden. Je nach den anzunehmenden Bezügen ändert sich natürlich die Bedeutung der Konstruktion. Irgendwie soll differenziert werden. Damit wird die grundsätzliche verfassungsrechtliche Zulässigkeit vorausgesetzt.

\subsection{Schwache Intension}

Ein in der Schlussphase der schwarz-roten Koalition auffällig unauffälliges Regierungsmitglied ist Guido Westerwelle (2011). Bei seiner letzten Regierungserklärung zu Afghanistan vor dem Bundestag entsteht der Eindruck, dass er nicht viel zu sagen hat. Sie schließt wie folgt:

Was wir in Afghanistan tun, tun wir für die Kinder, wir tun es natürlich auch für unsere eigene Sicherheit.

Nichts ist einfach in Afghanistan, und vieles ist noch nicht so, wie es sein soll. Ich fürchte, vieles wird auch schwierig bleiben. Aber am Ende dieses Jahres, nach der Bonner Konferenz, bin ich überzeugt: Wir sind mit unserem Einsatz und mit der neuen Partnerschaft auf dem richtigen Weg. Wir eröffnen Afghanistan die Chance auf eine friedliche und freie Zukunft - im Interesse der Menschen dort und im Interesse der Sicherheit hier.

(Westerwelle 2011)

Die Rede ist voller Floskeln, die wenig bis gar nichts aussagen. Für "Afghanistan" könnte ebenso gut "Europa" eingesetzt werden (oder eine beliebige andere Region, z. B. Afrika), und die Aussage wäre genauso treffend. Dass diese Floskeln fast immer passen, liegt daran, dass in dem Textausschnitt Indefinitpronomina (nichts, vieles) und relativ bedeutungsleere Substantive (Einsatz, Partnerschaft, Chance, Zukunft, Menschen) verwendet werden. Die Passepartout-Wörter haben jedoch alle eine positive Konnotation. Somit gelingt es dem Redner, im Unklaren zu verharren, dabei jedoch ein positives Gefühl zu vermitteln.

Hier zeigt sich noch einmal das wichtigste Merkmal von "Nebelsprech": Klare und bedeutungsschwere Aussagen werden vermieden - insbesondere das Nennen von verantwortlich Handelnden, die in Regress genommen werden könnten. Stattdessen überwiegen bedeutungs- 
leere (intensionsarme) Wörter (bzw. Wörter mit großer oder offener Extension wie die Pronomina wir und man) und Floskeln, die oft emotional aufgeladen sind (Konnotation) bzw. in einen positiven semantischen Kontext gesetzt werden (Framing). Dabei sind es gerade Konnotationen und Framing, die verräterisch sind, denn sie zeigen, welche Einstellung der Redner zu seinem Thema hat.

Für die Frage nach dem Zusammenhang von Sprache und Demokratie sind folgende Aspekte wichtig: Die parlamentarische Demokratie besteht zum einen im Werben für die eigene Position, was sich sprachlich in Anleihen an Werbestrategien niederschlägt, nämlich bei der Verwendung positiv konnotierter Wörter und Hyperbeln, sowie durch geschicktes Framing von zum Teil problematischen Konzepten. Zum anderen beruht die Demokratie auch auf dem Prinzip der Verantwortung. Das führt dazu, dass Politiker Versprachlichungsstrategien bevorzugen, die die eigene Regresspflicht mildern, indem sie auf Ungenauigkeit und Vernebelung abzielen, wie sich an den in diesem Beitrag diskutierten Beispielen zeigen lässt.

\section{Literatur}

Biermann, Kai/Haase, Martin (2012): Sprachlügen: Unworte und Neusprech von "Atomruine" bis "zeitnah". Frankfurt a. M.: Fischer.

Bird, Steven/Klein, Ewan/Loper, Edward (2009): Natural Language Processing with Python - Analyzing Text with the Natural Language Toolkit. Sebastopol, Cal.: O'Reilly.

Klemperer, Victor (1947): LTI - Lingua Tertii Imperii. Notizbuch eines Philologen. Leipzig: Reclam.

Lakoff, George (2014): Don't Think of an Elephant! Know Your Values and Frame the Debate. 2. Auflage. White River Junction: Chelsea Green Publishing.

\section{Korpusverzeichnis}

Bundesministerium der Finanzen (Hrsg.) (2009): Prinzip Rettungsschirm. www.bundesfinanzministerium.de/Content/DE/Standardartikel/Themen/Internationales_Fi nanzmarkt/Finanzmarktpolitik/076_Prinzip_Rettungsschirm.html [23.02.2015].

CDU/CSU-Fraktion (2011): Für den Euro - für Europa. http://eurorettungsschirm.cducsu.de/ [23.02.2015].

Deutscher Bundestag (Hrsg.) (19.01.2010): Plenarprotokoll 17/14. Stenographischer Bericht. Berlin: Deutscher Bundestag.

Deutscher Bundestag (Hrsg.) (2012): Breite Mehrheit für Fiskalpakt und Rettungsschirm. www.bundestag.de/dokumente/textarchiv/2012/39684652_kw26_de_fiskalvertrag_esm/20 8972 [23.02.2015].

Di Lorenzo, Giovanni (2011): "Es war kein Betrug". (Interview mit Karl-Theodor zu Guttenberg). www.zeit.de/2011/48/DOS-Guttenberg/ [23.02.2015].

Die Grünen Berlin (2011). Berlin ökologisch und sozial gestalten. Depubliziert. http://grueneberlin.de/sites/default/files/gemeinsam/LDK/ldk_20110930_k1.pdf [15.12.2011].

Gruner und Jahr (Hrsg.) (06.12.2012): Pressedatenbank. www.pressedatenbank.guj.de /PDB/Home.htm [06.12.2012].

Guttenberg, Karl-Theodor zu (2011): Guttenberg: "Es wurde allerdings zu keinem Zeitpunkt bewusst getäuscht". www.saarbruecker-zeitung.de/sz-berichte/themen/Guttenberg-Es- 
wurde-allerdings-zu-keinem-Zeitpunkt-bewusst-getaeuscht;art2825,3640829\# [23.02.2015].

Merkel, Angela (2011): Pressestatement zu Fukushima. www.bundeskanzlerin.de/Content/ DE/Mitschrift/Pressekonferenzen/2011/03/2011-03-14-bkin-lage-japanatomkraftwerke.html [19.05.2011].

Merkel, Angela (2012a): Rede der Vorsitzenden der Christlich Demokratischen Union Deutschlands. Textfassung. $\quad$ www.kas.de/upload/ACDP/CDU/Reden/2012-12 parteitag_rede_merkel.pdf [23.02.2015].

Merkel, Angela (2012b): Rede der Vorsitzenden der Christlich Demokratischen Union Deutschlands. www.youtube.com/watch?v=kWiAWMFIcEk [23.02.2015].

Orwell, George (1949): 1984. London: Secker/Warburg.

SPD (2011): Ordentlicher Parteitag: Beschluss 66: "Datenschutz und Grundrechte stärken Datenspeicherung begrenzen!". Www.spd.de/linkableblob/21948/data/66_beschluss _datenspeicherung.pdf [23.02.2015].

Westerwelle, Guido (2011). Eigenverantwortung und Partnerschaft - eine neue Perspektive für Afghanistan. www.auswaertiges-amt.de/DE/Infoservice/Presse/Reden/2011/111215 _BM_BT_AFG.html [23.02.2015]. 